\title{
Ciencia y tecnología para el desarrollo social en el contexto local. Experiencias de una Universidad
}

\section{Science and technology for social development in the local context. Experiences of a university}

\author{
Lic. Anays Martin Hernández ${ }^{1}$ \\ amhernandez@ucf.edu.cu \\ https://orcid.org/0000-0003-4241-5207 \\ MSc. Ania Odalis Hernández Aguila ${ }^{2}$ \\ oaguila@ucf.edu.cu \\ https://orcid.org/0000-0002-6594-0023
}

Recibido: 1/11/2020, Aceptado: 1/11/2020

\section{RESUMEN}

El presente trabajo aborda como la universidad constituye una institución que desde el cumplimiento de sus propias funciones hace posible el indisoluble nexo entre Ciencia Tecnología y Sociedad. A partir de un análisis de los resultados alcanzados por el Centro Universitario Municipal de Cruces en diferentes carreras y por la inserción en proyectos de investigación en el territorio, se ejemplifica como la utilización de la ciencia y la tecnología pueden ser puestas en función del desarrollo social en el contexto local.

Palabras clave: ciencia, tecnología, sociedad, universidad, contexto local.

\section{ABSTRACT}

The present work addresses how the university constitutes an institution that, from the fulfillment of its own functions, makes the indissoluble nexus between Science Technology and Society possible. From an analysis of the results achieved by the Municipal University Center of Cruces in different careers and by the insertion in research projects, it is exemplified how the use of science and technology can be put in function of social development in the local context.

Keywords: science, technology, society, university, local context.

1 Centro Universitario Municipal Cruces. Universidad de Cienfuegos Carlos Rafael Rodríguez. Cuba.

2 Universidad de Cienfuegos "Carlos Rafael Rodríguez", Cuba. 


\section{Introducción}

Los estudios acerca de ciencia, tecnología y sociedad son relativamente jóvenes. A lo largo de los años diversos autores han tratado sobre este tema. Muchos le han brindado mayor importancia a la ciencia clasificando a la tecnología como ciencia aplicada, y a la sociedad le dan un papel secundario. Sin embargo, la realidad ha demostrado que los tres constructos constituyen elementos que se encuentran íntimamente ligados y son una importante área de trabajo en investigación académica, política pública y en la educación.

En este sentido la universidad juega un papel trascendental, pues es la encargada de formar a los profesionales que llevarán a cabo el desarrollo científico tecnológico de un país garantizando, además, su superación permanente pero también, desde estas instituciones se llevan a cabo por estudiantes y profesores trabajos de investigación a partir de las necesidades sociales repercutiendo en el desarrollo de las localidades.

En Cuba, a partir del proceso de universalización se crea en cada municipio del país un Centro Universitario Municipal (CUM) como célula de la enseñanza superior en el territorio y por tanto desde entonces, se comenzaron a identificar las necesidades sociales que requerían de la gestión del conocimiento y la innovación para su solución.

El presente trabajo tiene como propósito ofrecer algunos elementos de cómo el CUM del municipio de Cruces, haciendo uso de la ciencia y la tecnología influyen en el desarrollo social de la localidad.

\section{Metodología}

La ciencia constituye un constructo que ha sido abordado a través de los años por disímiles autores dentro de los cuales se encuentra: F. Bacon y J. S. Mill; J. Herschel y W.S. Jevons; T. Kuhn, P. Feyerabend, N. R. Hanson, S. Toulmin o W. Quine; Barry Barnes, David Bloor o Steve Shapin; Bruno Latour y Steve Woolgar.

Las primeras ideas que hicieron referencia al tema planteaba que esta era una empresa autónoma, objetiva, neutral y basada en a aplicación de un código de racionalidad ajeno a cualquier tipo de interferencia externa (condicionantes sociales, políticos, psicológicos...), aquí se destacan los trabajos realizados por $\mathrm{F}$. Bacon y $\mathrm{J}$. S. Mill citado en Marino. E (2001).

Estos autores consideraban que el método científico era entendido básicamente como un método inductivo para el descubrimiento de leyes o fenómenos. Ya en el siglo XX, se ha producido una implicación creciente de la ciencia en la formulación 


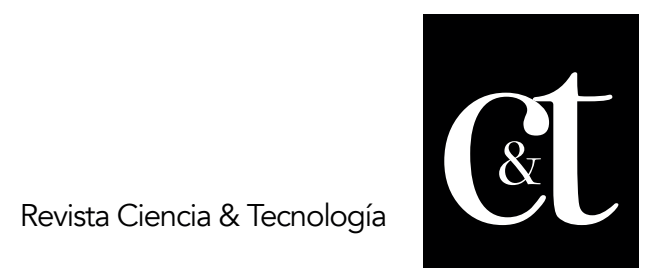

No. 29, 31 de enero de 2021

ISSN impreso: 1390 - 6321

ISSN online: 2661 - 6734

de políticas públicas ello ha conducido a la aparición de una actividad científica con características particulares.

Un elemento importante a tener en cuenta para estudiar la ciencia lo constituye el concepto de tecnología, el cual ha sido abordado por diferentes autores. En este sentido Marino. E (2001) la define como colección de sistemas diseñados para realizar alguna función.

Este autor siguiendo los trabajos de Radder (1996), plantea que hay cinco características claves que distinguen a la tecnología: debe ser realizable, tiene un carácter sistemático, pues está insertada en un entramado sociotécnico que la hace viable, los sistemas tecnológicos realizados son heterogéneos, mantiene una amplia y diversa relación con la ciencia, y crea relaciones de dependencia entre los diferentes agentes implicados.

En los últimos años se está hablando de estudios sobre la ciencia, la tecnología y también incluyen la sociedad, pues los tres factores se encuentran en una indisoluble estrechez.

En el caso del concepto de sociedad Marino. E (2001) aborda que, según los estudios realizados por la filosofía y la sociología, la sociedad es algo que procede de nuestra "naturaleza" pero que está sujeto a modificación y plantea que las sociedades se pueden definir por su desarrollo tecnocientífico, por su percepción de la tecnociencia y su relación con ella.

En este sentido entonces la ciencia, la tecnología y la sociedad (CTS) según el autor previamente citado, presentan como objeto de estudio los aspectos sociales de la ciencia y la tecnología, tanto en lo que concierne a los factores sociales que influyen sobre el cambio científico-tecnológico, así como las consecuencias sociales y ambientales que se derivan de ello.

Con respecto a este aspecto Núñez. J y Figaredo. F (2003) plantean que "se hizo cada vez más claro que la ciencia y la tecnología son procesos sociales profundamente marcados por la civilización donde han crecido; el desarrollo científico y tecnológico requiere de una estimación cuidadosa de sus fuerzas motrices e impactos, un conocimiento profundo de sus interrelaciones con la sociedad." Estos autores revelan que hoy en día los estudios CTS constituyen una importante área de trabajo en investigación académica, política pública y educación.

CTS define un campo bien consolidado institucionalmente en universidades, administraciones públicas y centros educativos de numerosos países industrializados y también de algunos de América Latina (principalmente Brasil, Argentina, México. Venezuela, Colombia y Uruguay). 


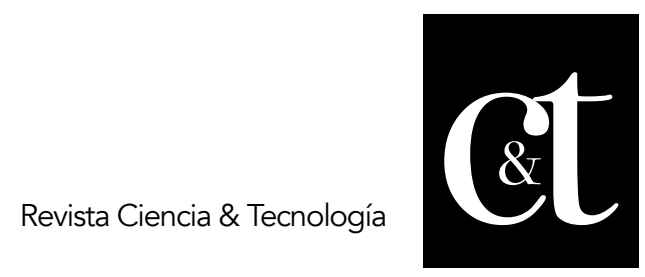

No. 29, 31 de enero de 2021

ISSN impreso: 1390 - 6321

ISSN online: 2661 - 6734

En Cuba, por su parte, desde inicios de la revolución en 1959 ocurrieron numerosas transformaciones sociales dentro de las cuales se pueden señalar la nacionalización de la enseñanza, el acceso gratuito a la educación, el desarrollo de la educación para adultos, los programas de enseñanza para campesinos y también la política científica y tecnológica (PCT). Esta última atravesó por diferentes etapas hasta llegar a lo que es hoy: "un país cuyo desarrollo científico, medido según los indicadores internacionales,

la sitúa en una posición favorable dentro de los países de América Latina y el Caribe (ALC)." Núñez. J y Figaredo. F (2003).

Según estos autores las principales características que presenta el sistema científico cubano son:

1. Educación terciaria de bastante buen nivel incluidas las ciencias y las ingenierías, así como avances en los niveles educativos precedentes que garantizan la promoción del talento que el avance de la ciencia necesita.

2. La formación de pregrado se continúa con un sistema nacional de postgrado capaz de formar investigadores, ofrecer educación permanente a sus graduados y de formar unos 400 doctores y 2000 maestros en ciencias y especialistas por año.

3. La educación en general y la preparación técnica de los trabajadores permiten una razonable capacidad de aprendizaje tecnológico de nuestras empresas.

4. El profesorado a dedicación completa tiene participación en actividades científicas orientadas a problemas de importancia local, regional, sectorial y nacional y ofrecen información y asesoría en tópicos relevantes de economía, salud, educación, agricultura, sociedad, medio ambiente, entre otros.

5. Uno de los desafíos que enfrenta la universidad en estos momentos es justamente la relación universidad - sociedad y más específicamente lo referente al desarrollo local (Coraggio. JL, 2002).

La Educación Superior Cubana desde las últimas cinco décadas ha desenvuelto su actividad y se ha desarrollado en un contexto de cambios sociales profundos que han tenido una notable influencia en la vida universitaria y en la relación de la Universidad con la sociedad. Esa relación Universidad-Sociedad, intensa, se revela en todas las funciones que desarrollan las instituciones universitarias en Cuba (formación profesional, postgrado, investigación y extensión universitaria). Las estrategias de formación e investigación universitarias se construyen en interacción con la sociedad. 


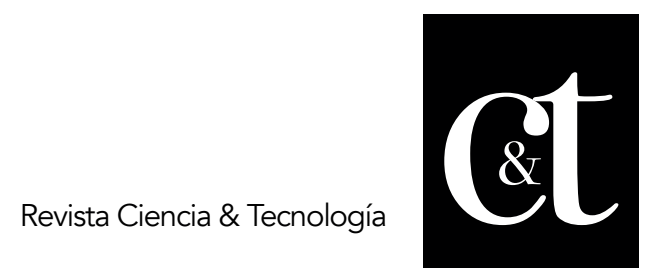

No. 29, 31 de enero de 2021

ISSN impreso: 1390 - 6321

ISSN online: 2661 - 6734

Una de las vías que favorecen este intercambio universidad - sociedad lo constituye las actividades que realizan las universidades para promover el desarrollo local. Según Coraggio. JL (2002) el desarrollo local "constituye el desarrollo de una economía, una sociedad y un sistema político locales, condiciones básicas para una mejoría sostenida en la calidad de vida de sus ciudadanos".

En función de esto la universidad puede contribuir a ofrecer un espacio público pluralista, convocando a todos los sectores a tratar de manera transparente los problemas de la sociedad local en el contexto nacional. Es por ello el sistema educativo y el de comunicación social deben transformarse, y la universidad puede jugar un papel de soporte de esas transformaciones, trabajando junto con los establecimientos secundarios, primarios, de educación inicial y de educación continua, y con los medios de comunicación de masas, como corresponsables de garantizar el derecho al conocimiento y la información de calidad para todos los ciudadanos.

Hoy en Cuba están constituidos los Centros Universitarios Municipales (CUM), los cuales fueron creados el 26 de noviembre del 2001 por el Acuerdo No. 6935 del Comité Ejecutivo del Consejo de Ministros. Estas instituciones integran los procesos universitarios que se desarrollan en los territorios, mediante mecanismos de coordinación, asesoramiento y control, para el incremento de la calidad y la pertenencia en materia de educación superior a nivel municipal en correspondencia con el desarrollo socioeconómico de cada municipio.

En el municipio de Cruces el CUM fue creado en el año 2002 con carreras tales como Derecho, Psicología y Estudios socioculturales y más adelante se fueron incorporando las carreras de Contabilidad y Finanzas, Ingeniería agronómica, ingeniería industrial, e ingeniería en Procesos Agroindustriales.

Por esta fecha se iniciaron también las primeras acciones de investigación de manera que se combinaran las actividades académicas, con las investigativas y la extensión universitaria y se llevaron a cabo, proyectos comunitarios en asentamientos poblacionales declarados como vulnerables por la dirección del territorio en los que se partió de un diagnóstico de la comunidad y se elaboraron acciones en coordinación con el gobierno y otros organismos que permitieron detectar problemáticas locales que no se conocían y dirigir acciones para su mitigación como eran actividades festivas, charlas, apertura de servicios que no existían, entre otras. En estas acciones participaron los estudiantes y profesores de diferentes carreras y dirigentes del gobierno y otras instituciones de la localidad y fue dirigido por profesores del CUM. 


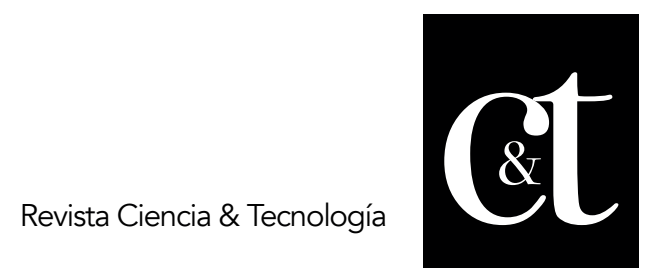

No. 29, 31 de enero de 2021

ISSN impreso: 1390 - 6321

ISSN online: 2661 - 6734

Por otra parte, en esa primera etapa, se inició un proyecto comunitario para el rescate de la cultura tradicional popular que permaneció por varios años, y entre sus principales resultados se encuentran el rescate de más de diez las tradiciones del municipio que ya estaban olvidadas las cuales quedaron ubicadas en un $C D$ en el museo de la localidad y la biblioteca como material de consulta para nuevas generaciones. El proyecto fue dirigido por una profesora a tiempo parcial quien era la historiadora del municipio y en el mismo participaron estudiantes y profesores de la carrera de Estudios Socioculturales.

Otro de los resultados más relevantes del centro donde se puede constatar la relación estrecha que existe entre la ciencia la tecnología y la sociedad lo constituyen las actividades llevadas a cabo a partir de la inserción de los profesores y estudiantes del centro en el Programa de innovación agrícola local (PIAL).

Aquí se establecieron redes que permitieron el intercambio y la interacción entre agricultores, técnicos, y otros actores, se crearon espacios que permitieron la socialización de experiencias, se planificaron y asesoraron el desarrollo de eventos científicos, profesores del CUM integraron el consejo científico de la agricultura para la retroalimentación de la situación que presenta el sector y rediseñar la estrategia del centro, si era necesario y se capacitaron agricultores y personal del sector en:

Manejo animal, montaje de celdas fotovoltaicas, utilización de biodigestores, entrenamiento sobre inseminación artificial.

Se desarrolla un sistema de conferencias sobre: Manejo agroecológico de plagas, Suelos y abonos orgánicos, el cultivo del frijol, alimento animal, el riego de agua, uso de las técnicas de riego.

También se impartieron temas jurídicos, se capacitan a juristas del sector en aspectos relacionados con la metodología de la investigación y se participa en un postgrado internacional sobre nutrición mineral en plantas y se multiplican los conocimientos en el territorio.

Por otra parte, se diagnostican trece fincas desde el punto de vista sociocultural y atendiendo a comercialización agropecuaria, silvicultura, extensionismo agrícola, sistema de producción y gestión económica y los resultados le fueron entregados a la agricultura constituyendo actualmente un documento de trabajo para ellos.

Se establece coordinación con otros proyectos existentes en el territorio. (Proyecto del arroz, proyecto Celia y Gestión Universitaria del conocimiento y la innovación para el desarrollo (GUCID) y agricultores del territorio y profesores de la filial participan en encuentros a diferentes niveles. 


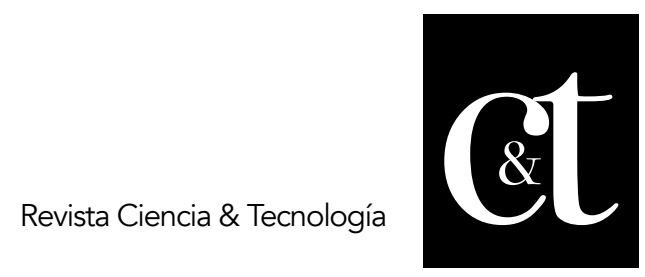

No. 29, 31 de enero de 2021

ISSN impreso: 1390 - 6321

ISSN online: 2661 - 6734

Por último a partir de este proyecto se introduce en el municipio la siembra del sagú como nuevo renglón agroalimentario y en coordinación con la estación experimental de la Universidad central de las Villas (UCLV) se montó un experimento que permite un estudio desde el inicio del cultivo hasta el análisis de los productos finales.

\section{Resultados y discusión}

Según el criterio de los directivos de las instituciones involucradas en el trabajo las acciones han contribuido a conocer mejor el contexto al quedar elaborados los diagnósticos de diferentes fincas con mayor profundidad y con la utilización de métodos científicos, ha sido posible identificar buenas prácticas y traerla al municipio así como socializar las nuestras al crear espacios con este propósito, se han identificado lideres no formales que permiten emprender nuevos proyectos y se han establecido redes con otras universidades, centros, especialistas y comunidades así como se ha logrado la integración de diferentes sectores que por muchos años se encontraban parcelados. También se ha incrementado la participación en eventos y las publicaciones relacionadas con diferentes temáticas.

Actualmente el CUM continúa llevando a cabo estas acciones y enriqueciéndolas con la experiencia práctica pero también se han incrementado las investigaciones pedagógicas pues estas son las carreras que en la actualidad predominan en el centro.

En este sentido se llevan a cabo estrategias que contribuyan a perfeccionar el desarrollo del proceso docente educativo en diferentes niveles de enseñanza, pero también las actividades extradocentes y comunitarias como parte del proceso de formación que se lleva a cabo en las escuelas del municipio.

\section{Conclusiones}

Los estudios CTS constituyen hoy un área importante de trabajo en investigación académica, política pública y educación y la universidad juega un papel trascendental pues todas las funciones que desarrollan las instituciones universitarias de formación profesional, postgrado, investigación y extensión universitaria se construyen en interacción con la sociedad.

Las acciones llevadas a cabo en el CUM del municipio de Cruces constituyen ejemplos de cómo la Ciencia y tecnología pueden contribuir al desarrollo social en el contexto local.

\section{Referencias}

Coraggio. JL (2002) Ponencia presentada en el Seminario Internacional.

La educación superior y las nuevas tendencias., organizado por el Consejo Nacional de Educación Superior (CONESUP), UNESCO y el CIESPAL, en Quito, 23-24 de julio 2002 


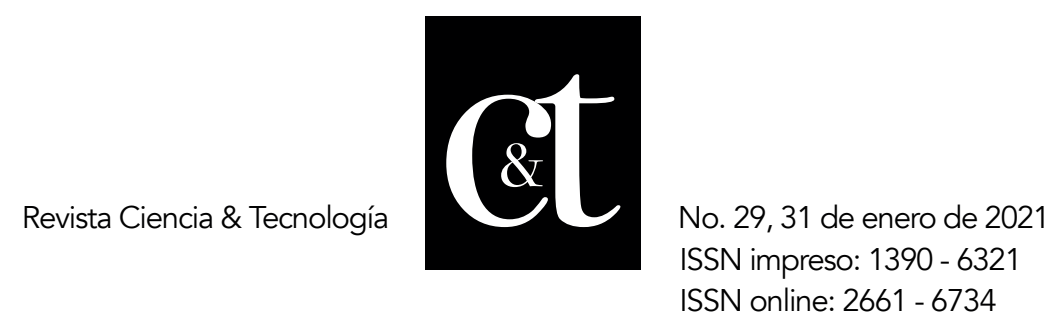

García-Guadilla, C (1997):"El valor de la pertinencia en las dinámicas de transformación de la educación superior en América latina", en La Educación Superior en el Siglo XXI. Visión de América Latina y el Caribe, Ediciones CRESALC/UNESCO, Caracas. Pp. 47-80.

Núñez. J (2007). Ciencia, Tecnología y Sociedad en Cuba: construyendo una alternativa desde la propiedad social.

. (2010) CTS en contexto: la construcción social de una tradición académica.

Núñez, J.; Montalvo, L.F.; Pérez, I.; Fernández, A.; García J. L. (2006): Universidad, innovación y sociedad: La Universidad cubana en el sistema nacional de innovación. Disponible en: http://developinguniversities.blogsome.com

Núñez, J. (1999/2001/2003): La Ciencia y la tecnología como procesos sociales. Lo que la educación científica no debería olvidar, Editorial Félix Varela, La Habana.

Marino. E (2001). Ciencia, Tecnología y Sociedad: una aproximación conceptual. Madrid.

Martín, Hernández. Ciencia y tecnología para el desarrollo social en el contexto local. Experiencias de una universidad 